\title{
Simultaneous Spinal and Intracranial Chronic Subdural Hematoma Cured by Craniotomy and Laminectomy: A Video Case Report
}

\author{
Hideki Kanamaru Kenji Kanamaru Tomohiro Araki Kazuhide Hamada \\ Suzuka Kaisei Hospital, Suzuka, Japan
}

\section{Key Words}

Simultaneous spinal and intracranial chronic subdural hematoma · Craniotomy ·

Laminectomy

\begin{abstract}
Simultaneous spinal and intracranial chronic subdural hematoma (CSDH) is a rare entity. A 67-year-old man visited our hospital due to headache after diving into a river 2 weeks before. Non-enhanced computed tomography (CT) and magnetic resonance imaging (MRI) revealed bilateral intracranial CSDH. The bilateral CSDH was evacuated and his symptoms improved. Three days after craniotomy, he complained of sensory disturbance on his buttocks. Lumbar MRI showed a space-occupying lesion behind the thecal sac at L5. CT with myelography showed a subdural mass lesion; there was no communication with the subarachnoid space. Fourteen days after craniotomy, L5 laminectomy was performed and the dura mater was incised carefully. The video shows that a liquid hematoma similar to the intracranial CSDH flowed out, followed by cerebrospinal fluid. His symptoms improved after the operation and the hematoma did not recur. This is a rare condition of spinal CSDH demonstrated by neuroimaging and intraoperative video.

(C) 2016 The Author(s)

Published by S. Karger AG, Basel
\end{abstract}

\section{Introduction}

Spinal subdural hematoma is rare entity with various etiologies that is related to anticoagulation, blood dyscrasia, lumbar puncture, trauma, spinal anesthesia or vascular malformation [1]. Simultaneous spinal and intracranial chronic subdural hematoma (CSDH) is

\section{KARGER}

Hideki Kanamaru, MD

Suzuka General Hospital

1275-53 Yamanohana, Yasuzukachou

Suzuka City, Mie Prefecture 513-0818 (Japan)

E-Mail hideki.k.722@gmail.com 
Kanamaru et al.: Simultaneous Spinal and Intracranial Chronic Subdural Hematoma Cured by Craniotomy and Laminectomy: A Video Case Report

extremely rare, with only 14 cases reported in the literature having undergone surgical evacuation for spinal lesions [2-12]. This surgical video case report demonstrates a patient suffering from simultaneous spinal and intracranial CSDH.

\section{Case Description}

History and Examination

A 67-year-old man visited the emergency department of our hospital because of nausea, headache and neck pain after diving into a river 2 weeks before. No abnormal neurological finding were present except for nausea, headache and neck pain. He had never taken antithrombotic medication or undergone lumbar puncture. Blood examination showed no bleeding tendency.

\section{Neuroimaging and Hospital Course}

On the day of admission, magnetic resonance imaging (MRI) showed bilateral intracranial subdural hematoma (fig. 1). On the same day, we performed bilateral craniotomy and the hematoma was evacuated. On day 4 after admission, the patient complained of sensory disturbance on his buttocks, and physical examination showed saddle anesthesia without other neurological abnormality, including bladder and rectal malfunction. On day 5, lumbar MRI demonstrated iso-signal intensity on T2-weighted imaging of a lesion similar to the intracranial subdural hematoma (fig. 2). On day 12, computed tomography (CT) with myelography demonstrated that the subdural hematoma extended from L4 to S1 (fig. 3). There was no communication between the lesion and the cerebrospinal cavity. The lesion effaced the thecal sac from L4 to S1. Cerebrospinal fluid examination showed hyperproteinorrhachia without blood contamination.

\section{Surgical Treatment for Spinal Subdural Hematoma}

On day 15 (27 days after he had dived), we performed L5 laminectomy and evacuated the subdural hematoma. It was liquid-like, similar to the intracranial CSDH. The hematoma flowed out followed by cerebrospinal fluid (video; see supplementary material at www.karger.com/doi/10.1159/000445709). We carefully inspected the inside of the dural sac, but could not find an apparent outer membrane, which is usually observed in intracranial CSDH. Neither abnormal vessels nor tumors were found. Finally the dura mater was closed.

After the operation, the patient's symptoms improved and lumbar MRI showed no acute recurrence. On day 38, he was discharged without neurological deficits. During 6-month follow-up no recurrence was observed.

\section{Discussion}

Spinal CSDH is a rare entity related to various causes [1]; most frequently it is spontaneous or related to minor trauma. In our case, there was no apparent direct impact other than diving into a river of several meters depth.

In the spinal subdural space, there are few vessels except that perforating the dura mater surrounding the neural root and the artery of Adamkiewicz found at the level of the thoracolumbar region. This may be the reason for the rarity of spinal subdural hematoma. Rader [13] described spinal subdural hematoma not caused by direct tearing of the bridging 
Kanamaru et al.: Simultaneous Spinal and Intracranial Chronic Subdural Hematoma Cured by Craniotomy and Laminectomy: A Video Case Report

veins, but by a rapid increase in the intravascular pressure of the lateral spinal arteries and veins.

On the other hand, Haines et al. [14] reported that there is no evidence of the subdural cavity as a natural state, but that the cavity originates from tearing of the dura-arachnoid junction, the so-called dural border cell layer. Through exposure to trauma or a disease process, fluids such as blood or cerebrospinal fluid will collect in the meninges. The pulsation of the cerebrospinal fluid may accelerate accumulations of blood by the 'water hammer like effect'. If this theory is the correct, the pathogenesis of spinal CSDH may be the same as that of intracranial lesions.

Some investigators demonstrated that spinal subdural hematoma might be caused by migration of the intracranial subdural blood [3]. Kokubo et al. [15] evaluated cases of intracranial CSDH by lumbar MRI, and only two cases presented with concomitant spinal subdural hematoma. They concluded that hematoma migration from the intracranial to the lumbar space was not possible.

In our case, MRI findings were similar for both the intracranial and the spinal lesion, and these findings mean that both hematomas occurred simultaneously. CT with myelography showed that the lesion did not move or communicate with the cerebrospinal space. These findings indicate the existence of an arachnoid membrane between the hematoma and the cerebrospinal space. During operation, after the incision of the dura mater, the hematoma flowed out immediately, followed by cerebrospinal fluid. These series of findings also indicate incision of the arachnoid membrane just after dural incision. The absence of a visible outer membrane could be the result of insufficient time to develop. The time needed for the development of the outer membrane of CSDH is still unclear, and it is possible that there is a difference in the speed of development between intracranial and spinal lesions.

\section{Conclusion}

We experienced a very rare case of simultaneous spinal and intracranial CSDH successfully treated by craniotomy and laminectomy.

\section{Statement of Ethics}

The authors have no ethical conflicts to disclose.

\section{Disclosure Statement}

The authors declare that they have no conflicts of interest to disclose.

\section{References}

1 Abla AA, Oh MY: Spinal chronic subdural hematoma. Neurosurg Clin N Am 2000;11:465-471.

2 Chen HJ, Liang CL, Lu K, Liliang PC, Tsai YD: Cauda equina syndrome caused by delayed traumatic spinal subdural haematoma. Injury 2001;32:505-507.

-3 Bortolotti C, Wang H, Fraser K, Lanzino G: Subacute spinal subdural hematoma after spontaneous resolution of cranial subdural hematoma: causal relationship or coincidence? Case report. J Neurosurg 2004;100(4 Suppl Spine):372-374. 
Kanamaru et al.: Simultaneous Spinal and Intracranial Chronic Subdural Hematoma

Cured by Craniotomy and Laminectomy: A Video Case Report

-4 Sari A, Sert B, Dinc H, Kuzeyli K: Subacute spinal subdural hematoma associated with intracranial subdural hematoma. J Neuroradiol 2006;33:67-69.

5 Moscovici S, Paldor I, Ramirez de-Noriega F, Itshayek E, Shoshan Y, Spektor S, Attia M: Do cranial subdural hematomas migrate to the lumbar spine? J Clin Neurosci 2011;18:563-565.

-6 Kirsch EC, Khangure MS, Holthouse D, McAuliffe W: Acute spontaneous spinal subdural haematoma: MRI features. Neuroradiology 2000;42:586-590.

7 Jimbo H, Asamoto S, Mitsuyama T, Hatayama K, Iwasaki Y, Fukui Y: Spinal chronic subdural hematoma in association with anticoagulant therapy: a case report and literature review. Spine (Phila Pa 1976) 2006;31: E184-E187.

-8 Lee JI, Hong SC: Spinal subdural haematoma as a complication of cranial surgery. Acta Neurochir (Wien) 2003;145:411-414; discussion 414-415.

-9 Yang MS, Tung YW, Yang TH, Chai JW, Chen CC, Chan SW, Tu CW: Spontaneous spinal and intracranial subdural hematoma. J Formos Med Assoc 2009;108:258-261.

10 Liu J, Wu B, Feng H, You C: Spinal subdural hematoma following cranial surgery: a case report and review of the literature. Neurol India 2011;59:281-284.

$\checkmark 11$ Ohta H, Ottomo M, Nakamura T: A case of the spinal subdural hematoma formation following ventriculoperitoneal shunting for von Recklinghausen's disease associated with aqueductal stenosis (in Japanese). No Shinkei Geka 2001;29:53-57.

-12 Cui Z, Zhong Z, Wang B, Sun Q, Zhong C, Bian L: Coexistence of spontaneous spinal and undiagnosed cranial subdural hematomas. J Craniofac Surg 2015;26:e118-e119.

13 Rader JP: Chronic subdural hematoma of the spinal cord: report of a case. N Engl J Med 1955;253:374-376.

14 Haines DE, Harkey HL, al-Mefty O: The 'subdural' space: a new look at an outdated concept. Neurosurgery 1993;32:111-120.

-15 Kokubo R, Kim K, Mishina M, Isu T, Kobayashi S, Yoshida D, Morita A: Prospective assessment of concomitant lumbar and chronic subdural hematoma: is migration from the intracranial space involved in their manifestation? J Neurosurg Spine 2014;20:157-163.

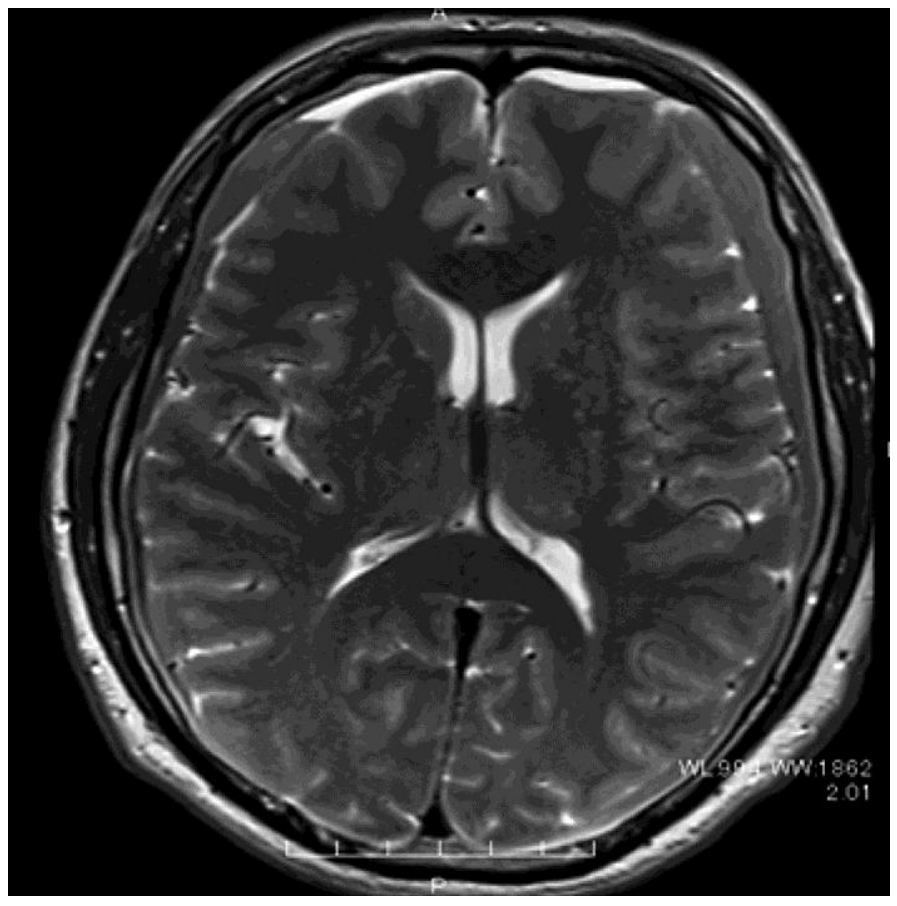

Fig. 1. Preoperative MRI (T2-weighted imaging) showed bilateral iso-signal intensity of the lesions. 
Case Reports in
Neurology

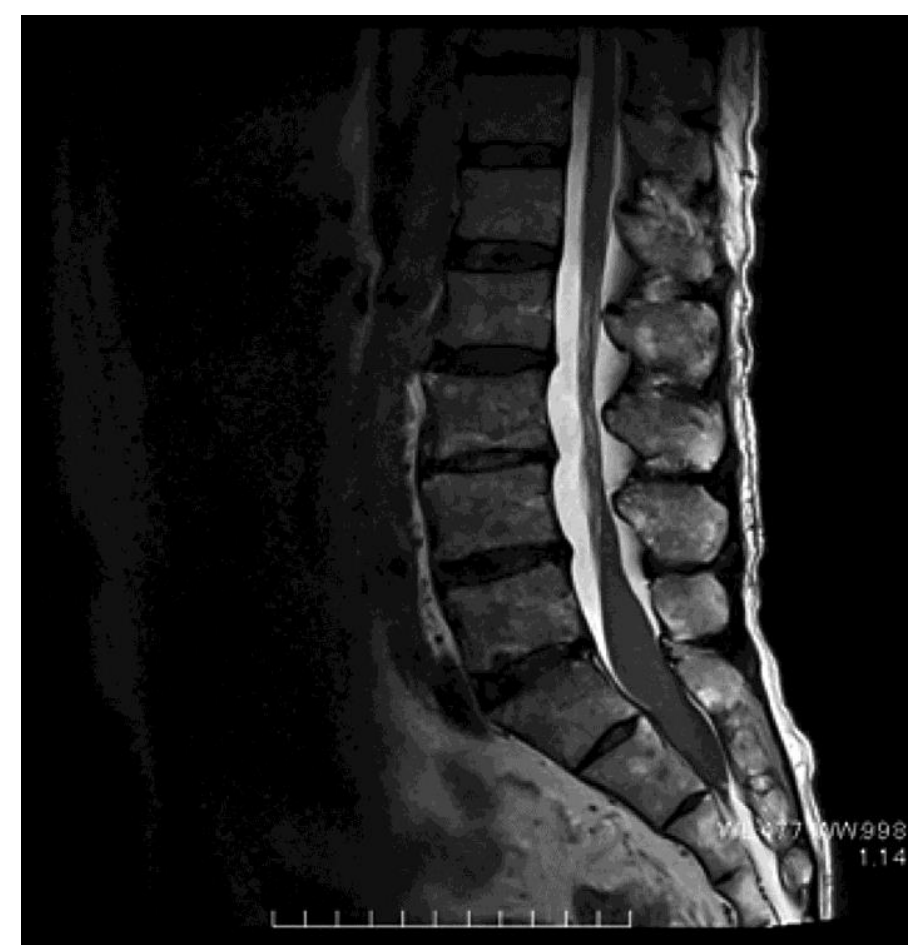

Fig. 2. Lumbar MRI (T2-weighted imaging) showing iso-signal intensity of the lesion similar to that of the intracranial subdural hematoma.

Case Rep Neurol 2016;8:72-77
DOI: $10.1159 / 000445709$

(c) 2016 The Author(s). Published by S. Karger AG, Basel www.karger.com/crn

Kanamaru et al.: Simultaneous Spinal and Intracranial Chronic Subdural Hematoma Cured by Craniotomy and Laminectomy: A Video Case Report 
Kanamaru et al.: Simultaneous Spinal and Intracranial Chronic Subdural Hematoma Cured by Craniotomy and Laminectomy: A Video Case Report

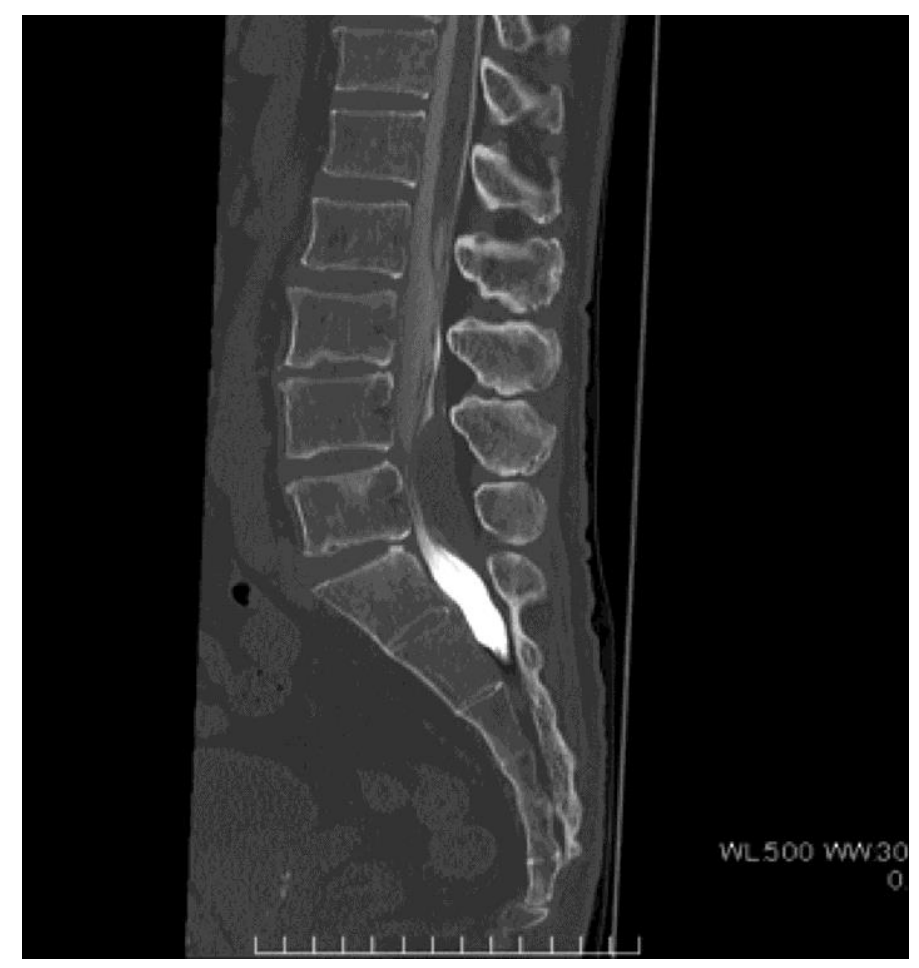

Fig. 3. CT with myelography showed that the lesion effaced the thecal sac; there was no communication with the cerebrospinal cavity. 